\title{
EL CARÁCTER SINTÉTICO DE LA LENGUA LATINA Y SU LEGADO CULTURAL
}

Tomás Jiménez Juliá*

\begin{abstract}
This paper deals with the possibility of establishing some kind of relationship between those languages which tend towards synthetic syntactic structures (not morphological) and speech communities with social and civil priorities which are equally synthetic and practical, and those languages with a more analytic syntax and communities with more 'analytic' civil and cultural priorities. The Roman world and Latin, as well as the British Empire and Modern English, seem to fit in the first frame, whereas Romance languages and European medieval societies, namely Spanish, could illustrate the second.
\end{abstract}

Keywords: typology, social idiosyncrasy, cultural legacies, synthetic syntax, analytic syntax.

Palabras clave: tipología, idiosincrasia social, legados culturales, sintaxis sintética, sintaxis analítica.

1.

\section{Síntesis y análisis. Nociones generales}

1.1. Síntesis, del griego $\sigma u ́ v \theta \varepsilon \sigma \iota \varsigma$, implica combinación, composición de un todo a partir de unas partes, en suma, 'unión', mientras que análisis, del griego, ảvá $\lambda v \sigma ı$, , implica lo contrario: separación

\footnotetext{
* Universidad de Santiago de Compostela, Facultad de Filología, Departamento de lengua y Literatura espańolas, Teoría de la literatura y Lingüística general, 15705 Santiago de Compostela, España, tomaseduardo.jimenez@usc.es.
} 
de un todo en parte1. Sintetizar es unir, condensar y quedarse con lo relevante, mientras que analizar es separar, descubrir las partes constitutivas de un todo y determinar su composición. Ambas operaciones son necesarias para el conocimiento, y, dependiendo del tipo de objeto de estudio prevalecerá una u otra: el análisis es necesario para averiguar la composición de algo que se nos muestra globalmente. La síntesis, para ver con claridad el valor de un conjunto que se presenta disgregado.

En el uso actual de los términos, y aplicado a personas o colectivos humanos, decimos que una mente sintética es aquella capaz de extraer lo relevante de entre un conjunto de elementos, 'juntando' adecuadamente lo importante. Una mente analítica, por su parte, es aquella que sabe descomponer un todo en sus partes, profundizando en ellas más que en el conjunto que forman. Todos conocemos gente muy analítica, rasgo que a veces va asociado a un exceso de susceptibilidad, y gente con gran capacidad de síntesis, que suele condensar rápidamente lo fundamental de las situaciones y dejar a un lado lo superfluo o prescindible. La tendencia a una u otra operación intelectual en la búsqueda del co-

1 Los diccionarios de griego traducen el término ảvó $\lambda \cup \sigma \iota \varsigma$, deriva-

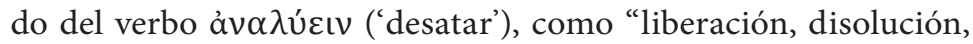
fin, muerte. Solución” (Pabón, 1967, s.v.). Por su parte, el término $\sigma u ́ v \theta \varepsilon \sigma \iota \varsigma$ se refiere a "combinación, arreglo, mezcla; reunión, composición [...]” (idem). El castellano adoptó estas palabras, de registro culto o técnico, con los significados de "Resolución de una cosa a sus principios” (Real Academia Española, 1726, s.v. Analysis) y de "Lo mismo que composición" (idem s.v. Synthesis). Actualmente las definiciones del DRAE (versión en línea: http:// dle.rae.es/?w=diccionario) no se apartan mucho de éstas, aunque se precisan más: Así, las dos primeras acepciones para 'análisis' son: 1. Distinción y separación de las partes de algo para conocer su composición; 2. Estudio detallado de algo, especialmente de una obra o de un escrito.

Por su parte, 'síntesis' tiene como primera acepción "Composición de un todo por la reunión de sus partes”. Las primeras acepciones de sendos términos son las que se usan para caracterizar las lenguas como (de tendencia) 'analítica' o 'sintética', y las que utilizaré en general aquí. 
nocimiento, tanto práctico como científico, no es algo individual necesariamente: a menudo puede verse en las comunidades una cierta tendencia hacia una forma más analítica o más sintética de contemplar la realidad y buscar conocimientos y soluciones diversas, lo cual también puede llegar a reflejarse en el modo de expresar la información.

Antes de continuar vea mos muy rápidamente cómo se han reflejado estas tendencias a la síntesis o al análisis en las lenguas.

1.2. Como ya apunté en otro lugar (Jiménez Juliá, 2017a: $\$ 1$ ), la caracterización de una lengua como (de tendencia mayoritariamente) analítica o sintética sigue haciéndose hoy día del mismo modo que cuando se acuñó hace dos siglos (Schlegel, 1818), esto es, apelando a la estructura morfológica de las palabras: de acuerdo con ello, las lenguas flexivas, que integran informaciones diversas como parte consustancial de la palabra (esp.: much-ísim-o-s; alem.: gesund-est-e; pol.: śpiewa-ł-by-m ...), o las aglutinantes, que, sin hacer que formen parte de ella, 'adhieren' información a la palabra (jap.: watashi-ni-wa; vasco: lurr-alde-ko-en-zat) se consideran 'sintética', porque las palabras, al menos las clases fundamentales (nombres, verbos) incluyen una información variada dentro o alrededor de sí. Por el contrario, las lenguas en las que la información no se integra en las palabras en forma de morfemas o elementos periféricos tendrán un carácter sintético (cfr. Trask, 1993: 15 y 273; Bussman, 1996: 57 y 1170; Siegel et al., 2014: 52-53). Se suele citar como ejemplo prototípico de lengua aislante y, por tanto, 'analítica', el chino.

La clasificación es, sin duda, interesante, pero el tiempo ha mostrado algunos problemas en su utilización para determinar la naturaleza de las lenguas. Uno de ellos, que nos interesa especialmente, es la inexistencia de lenguas tipológicamente puras y, por tanto, lo inadecuado de adscribir a una lengua un carácter globalmente sintético o analítico por el hecho de que el fenómeno estudiado se comportase de acuerdo con una tendencia determinada (cfr. Schwegler, 1990: 47 y ss.). Se puede hablar de mayor tendencia estadística en uno u otro sentido, pero 
difícilmente de comportamiento uniforme de una lengua a lo largo de toda su estructura morfológica. Ni siquiera el chino, prototipo de lengua analítica, carece de unidades calificables de sintéticas (cfr. Moreno Cabrera, 2003, que sigue a Her, 2002). Una segunda cuestión, que hace que la clasificación en cuestión no parezca suficiente para determinar la tendencia dominante (nunca única) de una lengua es, precisamente, su carácter estrictamente morfológico. La división entre lenguas o componentes de lenguas sintéticas y analíticas se ha centrado en si la información asociada a la palabra está incluida en ella (sintéticas) o fuera de ella (analíticas). No ha habido, que yo sepa, un intento de extender esta clasificación a ámbitos muy representativos de la naturaleza de las lenguas, como es el de la sintaxis ${ }^{2}$. En efecto, la sintaxis de una lengua nos dice el modo como se organizan los mensajes para expresar enunciados comunicativos y, por tanto, cuáles son nuestras tendencias constructivas a la hora de comunicarnos, de interaccionar unos con otros. Por ello, recientemente he propuesto extender el parámetro 'análisis-síntesis' a este campo, variando, naturalmente, el tipo de criterios que decide si una construcción sintáctica debe considerarse analítica o sintética en relación con lo que define ambos casos en morfología. Remito a Jiménez Juliá (2017a y 2017b) para mayores precisiones sobre este caso, y aquí me limitaré a señalar que en lo que se refiere a criterios específicamente sintácticos, la diferencia análisis-síntesis puede verse en hábitos constructivos que inciden, respectivamente, bien en la tendencia a pormenorizar la información en unidades diferentes o, por el contrario, en hacer construcciones compactas, 'económicas', que, con pocos medios den mucha información sin necesidad de elementos que

2 Hay caracterizaciones tipológicas con criterios sintácticos, como la conocida de Greenberg (1963), basada en el orden de constituyentes, o las que dividen las lenguas según su orientación nominativo-acusativa o ergativa (Dixon, 1994), pero no conozco estudios que se centren en la tendencia analítica o sintética de las lenguas según hábitos sintácticos. 
la atomicen. Así, la tendencia a la construcción analítica puede condensarse en el hábito de

a) desperdigar la información léxica en elementos diferenciados, y cuanto más independientes sean estos elementos, mayor carácter analítico (sintáctico) tendrá la lengua, y

b) explicitar las relaciones entre los elementos mediante recursos aislados más o menos especializados y de uso obligado.

Por el contrario, una lengua de tendencia sintácticamente sintética:

c) condensará la información en construcciones que omiten explicitar todos los componentes que derivan en la interpretación final, $y$

d) omitirá recursos que expliciten relaciones en la medida en que no exista ambigüedad.

De hecho, como ya hemos visto, analizar es 'separar', mientras que sintetizar es 'unir'. De acuerdo con estas premisas, una lengua puede tener unos rasgos morfológicos que la acerquen a una de estas tendencias, y unos rasgos sintácticos que la incluyan en la otra: Existe la posibilidad de que la lengua sea uniforme en sus tendencias tanto en su morfología como en su sintaxis, pero aún así habrá siempre en las lenguas componentes contradictorios con lo que puede ser su tendencia dominante, pues es este un parámetro complejo sujeto a muchas variables. El siguiente cuadro, puramente intuitivo, puede esquematizar las posibilidades teniendo en cuenta una serie de lenguas relevantes aquí3:

3 No hay que considerar el cuadro 1 como una descripción rigurosa y fundamentada de las tendencias tipológicas de las lenguas. Se trata de un cuadro intuitivo que ni ha utilizado métodos de medición cuantitativa (como los derivados de Greenberg, 1960) ni otro método calificable como científico. No tengo fuentes para establecer el 'grado' de analiticidad sintáctica de las lenguas porque no es este un concepto usado regularmente. Para situar la posición sintáctica del polaco dentro del esquema me han sido útiles las indicaciones que sobre la creación léxica en esta lengua y en español se encuentran en Sorbet (2017). 
Cuadro 1. Tendencias de las lenguas

\begin{tabular}{|c|c|c|c|c|c|}
\hline & \multicolumn{2}{|c|}{ Tendencia analítica } & \multicolumn{3}{|c|}{ Tendencia sintética } \\
\hline $\begin{array}{l}\text { MORFOLÓGICAMENTE } \\
\text { (estructura } \\
\text { de la palabra) }\end{array}$ & chino & inglés & $\begin{array}{l}\text { japonés } \\
\text { español }\end{array}$ & \begin{tabular}{|l} 
polace \\
latín
\end{tabular} & o protoindoeuropeo \\
\hline $\begin{array}{l}\text { SINTÁCTICAMENTE } \\
\text { (construcciones con } \\
\text { palabras) }\end{array}$ & chino & $\begin{array}{l}\text { español } \\
\text { japonés }\end{array}$ & polaco & $\frac{\text { latín }}{\mathrm{i}}$ & $\begin{array}{l}\text { protoindoeuropeo } \\
\text { inglés }\end{array}$ \\
\hline
\end{tabular}

Aunque no puedo detenerme en la justificación detallada de esta clasificación, podemos considerar que una lengua como el chino, por su ausencia de flexión o aglutinación es morfológicamente analítica, pero tampoco muestra signos de compresión sintáctica como vemos en latín, inglés o protoindoeuropeo. El español es morfológicamente sintético, aunque ya lejos de polaco o latín, sobre todo por la ausencia de flexión casual, pero sintácticamente es una lengua muy analítica, que presenta muy claramente los rasgos (a-b) antes apuntados. El comportamiento opuesto, al menos, en rasgos sintácticamente relevantes, es el del inglés: morfológicamente ha perdido la mayor parte de los rasgos que configuran una lengua sintética, pero sintácticamente muestra claramente los rasgos (c-d). El polaco, a modo de curiosidad, no muestra en absoluto los rasgos (c-d), por lo que no parece ser claramente sintética, pero su ausencia de artículo y de perífrasis y la escasa utilización de los pronombres personales unido a algunas construcciones comprimidas, averbales, como las 'Frases Nominales puras' del protoindoeuropeo (To nasza ulica: 'Esta es nuestra calle'), hacen que el resultado final tenga una apariencia realmente sintética ${ }^{4}$.

4 En efecto, si comparamos construcciones polacas con sus traducciones al inglés según el programa de enseñanza de lenguas duolingo, https:/es.duolingo.com/ [20.03.2017], podremos observar una multiplicación de elementos de las secuencias de esta última en relación con sus equivalentes polacas: we are looking for a tomato (szukamy pomidora); We do not have a bathroom here (Nie mamy tu łazienki); This is our street (To nasza ulica); A girl is eating (dziewczynka je) etc. 
Es evidente que la presencia necesaria en inglés de los pronombres personales sujeto, y la de determinantes articulares (indefinidos), unido al uso de formas verbales compuestas, hacen parecer esta lengua como sintácticamente analítica, sobre todo al lado de la compresión que muestra el polaco, con formas verbales sintéticas y diferenciadas aspectualmente que ahorran las expresiones aspectuales continuativas perifrásticas del inglés. El polaco, sin embargo, carece de las construcciones sintácticas directas (resultativas, phrasal verbs) características del inglés y que le otorgan ese carácter sintético tan peculiar de ciertas lenguas germánicas 5 .

Finalmente, el latín, que es la lengua que nos ocupa, presenta un carácter todavía marcadamente sintético en el campo morfológico, aunque ya en menor medida que el proto-indoeuropeo; sintácticamente, su tendencia puede verse igualmente como más inclinada hacia la síntesis que al análisis, pues aunque no explota, como el inglés, construcciones de tipo directo como las mencionadas en la nota 6 y utiliza ya las partículas con cierta obligatoriedad, carece de recursos analíticos como los desarrollados por las lenguas románicas para marcar o resaltar relaciones sintácticas, conserva, al menos en el latín literario, cierto uso de construcciones averbales (inteligentibus, pauca) y economiza medios (Mali et bona), además de omitir información redundante. Todo ello en relación con sus descendientes románicas.

Podemos considerar, por tanto, que las tendencias de una lengua -en última instancia, de los hablantes-a construir mensajes más condensados, sintéticos, y no tan pormenorizados o analíticos, puede reflejar mejor la idiosincrasia comunicativa de una comunidad que los modos de construir unidades morfológicas, más centradas en cómo se distribuye la información de unidades léxicas concretas.

5 Las construcciones resultativas son aquellas en las que mediante un predicativo que señala un resultado final se pueden expresar dos procesos mediante un solo verbo. El inglés he ate his plate clean supone el proceso de 'comer + dejar el plato limpio'. Las lenguas románicas carecen de este recurso regular. Este esquema es el mismo de las llamadas phrasal verbs (Don't tell me off!), igualmente ajenas a las lenguas románicas. Sobre estas construcciones puede consultarse Mendívil Giró (2003) o Jiménez Juliá, Doval Reixa (2014). 


\section{Tipo de lengua y tipo de conducta}

2.1. Aunque sabemos que existen 'mentes' sintéticas y analíticas, y lenguas que siguen esas características, podemos preguntarnos si unas y otras están relacionadas de algún modo, esto es, si el hablante de una lengua de tendencia sintética tiende a llegar al conocimiento de las cosas a través de la síntesis -y lo mismo con el análisis-, o si no se puede establecer vinculación alguna al respecto. Sabemos que es peligroso, además de absurdo, establecer juicios de valor sobre las lenguas y sus hablantes basándonos en sus características tipológicas. En ese error cayó August Wilhelm von Schlegel (1818), identificando la estructura morfológica de las lenguas con la inteligencia colectiva de los hablantes ${ }^{6}$, y concluyendo que las lenguas clásicas, prototípico de las sintéticas, eran 'superiores'7. En términos valorativos, para establecer jerarquías entre las mentes de las comunidades hablantes según cuál sea la tipología de su lengua, esta asociación no tiene ninguna base ni científica ni apelando al mero sentido común. Otra cosa muy distinta es que las tendencias comunicativas estén en consonancia con otros hábitos intelectuales y culturales, y que las tendencias en el 'empaquetamiento' de la información puedan asociarse a las prioridades en otros órdenes de la actividad mental. Y ahí es donde se puede especular (de momento no voy más allá) sobre la relación entre análisis y síntesis 'sintáctica' y planteamientos culturales aparentemente concomitantes.

2.2. Es evidente que entre las distintas manifestaciones culturales de una comunidad suele haber, si no uniformidad, al menos cierta coherencia. Los estudios antropológicos, sobre todo los de corte es-

6 Para Schlegel, las lenguas sintéticas: “appartiennent à une autre phase de l'intelligence humaine: il s'y manifeste une action plus simultanée, une impulsion plus immédiate de toutes les facultés de l'âme que dans nos langues analytiques. A celles-ci préside le raisonnement, agissant plus à part des autres facultés, et se rendant par conséquent mieux compte de ses propres opérations" (Schlegel, 1818: 27-28).

7 "Je l'avoue, les langues anciennes, sous la plupart des rapports, me paroissent bien supérieures” (Schlegel, 1818: 25). 
tructural (Radcliffe-Brown, 1952; Lévi-Strauss, 1958, o, incluyendo más explícitamente la lengua, Malinowski, 1923) habían puesto de manifiesto en su momento algo que hoy día es un axioma de la antropología, a saber, la existencia de una interrelación entre las distintas actividades asociadas a una cierta cultura. La cuestión, que se ha debatido desde siempre es si algo tan específico como la lengua es un reflejo de la mentalidad de sus hablantes o es algo que está más allá de ellas. Y también en sentido inverso, si es la lengua la que condiciona la visión del mundo. La idea de la conformación de la mentalidad de la comunidad según la lengua que se hable es lo que ha dado lugar al conocido como 'relativismo lingüístico'. En su formulación más extrema (Whorf, 1956), el relativismo no parece demasiado creíble, pues la forma de ver el mundo de una comunidad es el resultado de la confluencia de muchos parámetros, y darle el peso que se le daba a la lengua en este aspecto no parece empíricamente justificado, pero ello no obsta para que pueda haber aspectos parciales de la lengua que tengan una estrecha relación con otros relativos a la idiosincrasia de la comunidad que la habla. Aquí voy a partir de la base de que, sin hacer juicios apriorísticos, es posible constatar empíricamente coincidencias entre algunos aspectos de la lengua y algunos aspectos de la sociedad en la que se ha ido conformando. Dado que el tema que me ocupa en este momento es el relativo al carácter analítico o sintético de las lenguas, trataremos de ver si podemos establecer algún tipo de relación entre la naturaleza (prioritariamente) sintética o analítica de una lengua y las formas dominantes de acceso al conocimiento (mediante síntesis o análisis) y otras características asociadas en la comunidad que la habla 8 .

8 Insisto en que dejo a un lado la inconveniencia de considerar que una lengua caracterizada como analítica o como sintética lo es en todas sus manifestaciones. Todas las lenguas tienen aspectos contradictorios en distintos componentes: pueden ser analíticas en el verbo y sintéticas en las construcciones nominales, o analíticas morfológicamente y sintéticas sintácticamente. Aquí consideraremos una lengua como de tendencia sintética o analítica según la existencia de construcciones sintácticamente relevantes en uno u otro sentido. 
2.3. Antes de entrar en el carácter más o menos sintético o analítico de una comunidad histórica (como el mundo romano), conviene hacer una pequeña reflexión sobre nuestro modo de encarar el tema. Cuando hacemos un juicio o, incluso, una mera descripción, de hechos complejos, como pueden ser grandes culturas o movimientos culturales concretos, períodos o acontecimientos históricos, nos quedamos obviamente con lo relevante, haciendo una abstracción de aspectos que no interesan, y el resultado es que tenemos una imagen e, incluso, una valoración, de estos hechos, movimientos o períodos muy filtrada por esa valoración. Con mucha frecuencia la imagen proyectada es una reconstrucción posterior ajena a la conciencia que en su momento tenían los protagonistas de la historia: Hablamos de los Modistas medievales, pero sus componentes no tenían probablemente la conciencia de grupo homogéneo que la historia de la lingüística posteriormente les atribuiría. Y lo mismo con los miembros de la Generación del 98 o del Movimiento cubista. Todas estas etiquetas son 'abstracciones' que hace alguien a posteriori y que, a veces incluso chocan con la visión de los protagonistas sobrevivientes.

Estas abstracciones, cuando implican juicios históricos, no meras descripciones o interpretaciones artísticas o culturales, se hacen desde la óptica dominante en el momento: tras un conflicto la descripción del mismo que prevalece es la que hacen los vencedores. De ahí que cuando cambia la correlación de fuerzas, haya 'revisiones' de la historia. Con ejemplos concretos, el Descubrimiento de América fue una gesta heroica, pacífica y enriquecedora, cuando la describe la oficialidad española, pero cuando los países hispanoamericanos empiezan a propagar su visión y tienen posibilidad de ser tenidos en cuenta, la gesta se convierte en expolio, explotación y genocidio. Lo mismo podemos decir de hechos como la Revolución rusa o la Guerra civil española.

Para objetivar ciertas cosas, a la hora de juzgar o valorar un hecho, un período o una cultura, podemos fiarnos del legado que nos ha dejado, esto es, lo que hemos obtenido las generaciones o períodos históricos posteriores del paso de esta cultura o fuer- 
za dominante en una cierta época. El juicio del legado, naturalmente, también está sujeto a muchas matizaciones, pero para lo que aquí nos interesa podemos simplificar y considerar que los legados pueden dividirse en dos grandes tipos: los que nos dejan planteamientos generales en ciertas parcelas de nuestra vida intelectual o práctica, y los que nos dejan desarrollos más concretos, producto del planteamiento hecho por otros. Esto es, de un modo simplificado podríamos decir que hay legados más intelectuales y otros más prácticos. Pero tampoco esta división sería exacta, pues dentro de los planteamientos generales, esto es, los legados 'intelectuales', los hay encaminados a solucionar problemas verdaderamente prácticos. Por eso, y con el fin de ahorrar tiempo, podemos guiarnos por el cuadro 2 donde vemos distintas posibilidades de caracterizar legados culturales, así como ejemplos, a modo de mera ilustración, y con todas las prevenciones posibles, que he considerado apropiados para cada caso.

Cuadro 2. Legados culturales

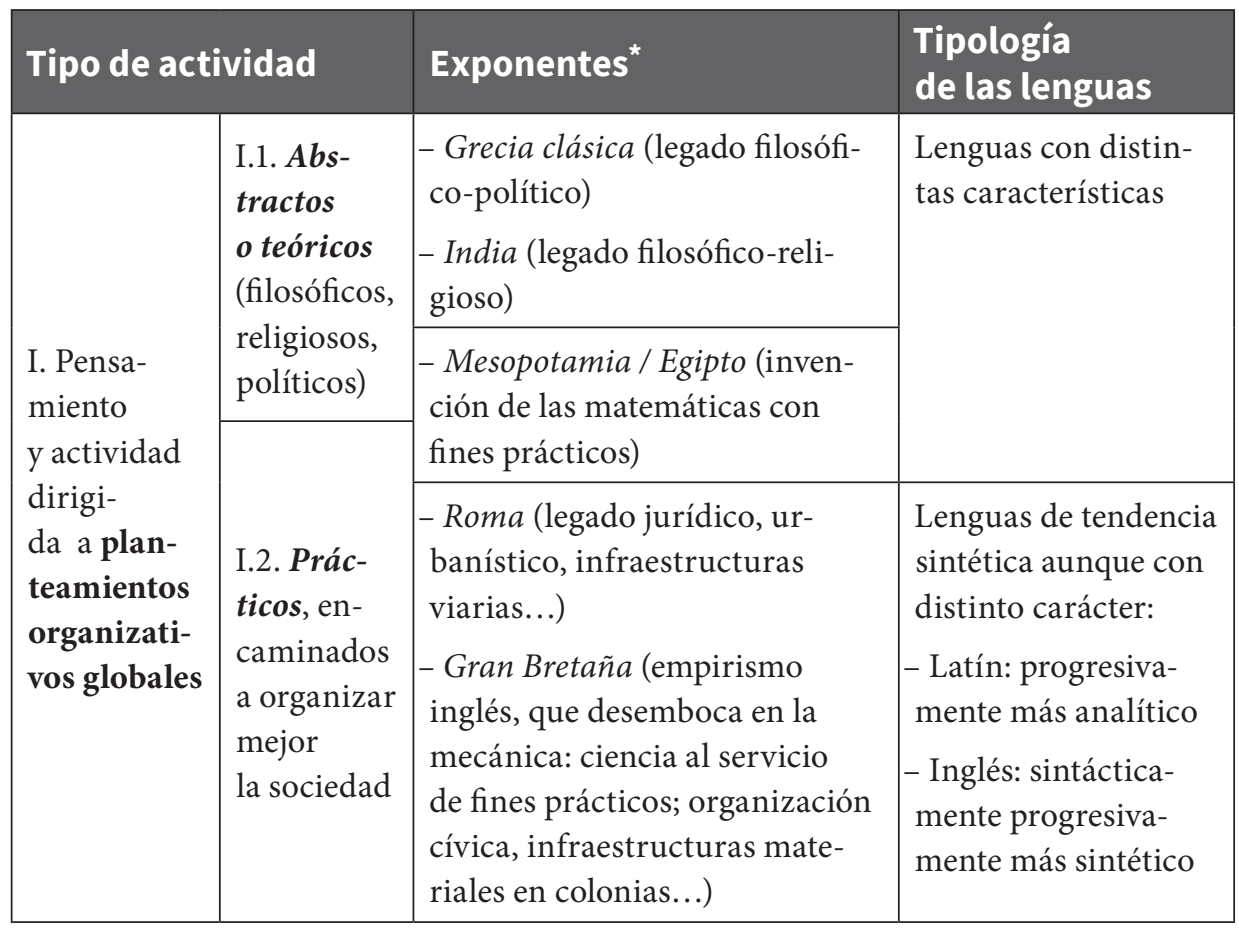




\begin{tabular}{|l|l|l|}
\hline Tipo de actividad & Exponentes $^{*}$ & $\begin{array}{l}\text { Tipología } \\
\text { de las lenguas }\end{array}$ \\
\hline \multirow{2}{*}{$\begin{array}{l}\text { II. Desarrollo y profun- } \\
\text { dización en planteamien- } \\
\text { tos previos }\end{array}$} & $\begin{array}{l}\text { - Sociedades medievales pos- } \\
\text { tromanas y proto-románicas } \\
\text { (exégesis del pensamiento gre- } \\
\text { co-romano y cristiano; desarro- } \\
\text { llo de artes previas, sin grandes } \\
\text { innovaciones) }\end{array}$ & $\begin{array}{l}\text { Lenguas globalmente } \\
\text { progresivamente más } \\
\text { analíticas }\end{array}$ \\
\cline { 2 - 3 } & $\begin{array}{l}\text { - Japón moderno: desarrollo } \\
\text { tecnológico y profundización } \\
\text { en planteamientos a menudo } \\
\text { ajenos }\end{array}$ & $\begin{array}{l}\text { Lengua morfológi- } \\
\text { camente sintética } \\
\text { (aglutinante), sintác- } \\
\text { ticamente con rasgos } \\
\text { analíticos y con un } \\
\text { sistema de escritura } \\
\text { analítico }\end{array}$ \\
\hline
\end{tabular}

* Los exponentes son meros ejemplos del legado universal de ciertas culturas, adecuados para la comparación lingüística que quiero mostrar. En absoluto pretenden ser una visión completa o exhaustiva del papel de las distintas culturas milenarias en el avance de la humanidad. He dejado fuera, por ejemplo, a China, con su importante legado tanto en planteamientos filosóficos (el confucionismo y su influencia en Asia) como en inventos prácticos (bélicos: pólvora; culturales: papel; alimenticios: pasta; doméstico: seda...), o la astronomía maya o egipcia, pues no fueron legados inmediatos, sino logros culturales desconocidos por las demás culturas durante mucho tiempo.

De acuerdo con el cuadro, vemos la existencia de culturas cuya tendencia parece ser a (I) hacer planteamientos globales para organizar la vida social, frente a (II) la tendencia a utilizar planteamientos de otros y profundizar en ellos. Dentro de aquellas sociedades que crean planteamientos, esto es, dentro de (I), las hay más encaminadas a organizar la vida material, mundana (I.2), frente a los que tienen planteamientos más filosóficos o religiosos $(\mathrm{I} .1)^{9}$. Los primeros, particularmente los que tienden a buscar la

9 Sin relación con la lengua, un corte similar entre planteamientos espirituales y planteamientos prácticos es el que señala Edward Conze (1978) en su excelente introducción al budismo. Según Conze, Asia es el dominio de la espiritualidad, de la preparación para una vida extra-mundana, mientras que Occidente (básicamente, el occidente clásico) es el de la organización de la vida terrenal, debido ello a las diferentes posturas ante nuestra presencia en el mundo. En sus 
utilidad práctica desde el punto de vista material, prescindiendo de lo superfluo (I.2), suelen asociarse a sociedades que en otros parámetros muestran tendencias sintéticas, mientras que a menudo las sociedades incluidas en (II) suelen tener rasgos analíticos relevantes en muchas facetas.

\section{3.}

\section{Algunas ilustraciones}

En las líneas precedentes hemos visto básicamente dos cosas: (1) la existencia en las lenguas de tendencias constructivas, relevantes tanto en sus aspectos morfológicos como sintácticos, y (2) la existencia de prioridades en las culturas, detectadas a través de sus legados. Estos legados permiten ver las pautas seguidas en el quehacer de una determinada sociedad, bien centrándose en

palabras: "Con frecuencia se da por sentado que hay alguna diferencia fundamental entre Oriente y Occidente, entre Europa y Asia, en su actitud frente a la vida, en su sentido de los valores y en los procesos de sus almas. Los cristianos que piensan que el budismo no es apropiado para las condiciones de vida europea olvidan el origen asiático de su propia religión, y si a eso vamos, el de todas las religiones. Una religión es una organización de aspiraciones espirituales, que rechazan el mundo sensorial y niegan los impulsos que nos unen a él. Durante 3.000 años, solo Asia ha creado ideas y métodos espirituales. En estas cosas, los europeos han tomado prestado de Asia, han adoptado ideas asiáticas y, frecuentemente, las han vulgarizado. No creo que se pueda señalar en Europa ninguna creación espiritual que no sea secundaria, que no tenga su impulso último en Oriente. El pensamiento europeo se ha destacado en la elaboración de las leyes y la organización sociales, especialmente en Roma e Inglaterra, y en la comprensión y el dominio científicos de los fenómenos sensoriales. La tradición europea se inclina hacia la afirmación de la voluntad de vivir, y tiende a ir activamente hacia el mundo de los sentidos. La tradición espiritual de la humanidad está basada en la negación del deseo de vivir y mira en dirección opuesta a la del mundo de los sentidos. Toda la espiritualidad europea ha tenido que ser renovada periódicamente por algún influjo de Oriente, desde la época de Pitágoras y Parménides" (Conze, 1978, 12-13). 
planteamientos generales, sea con fines prioritariamente intelectuales o espirituales, o con fines prácticos, o bien prescindiendo de planteamientos propios y profundizando en marcos heredados, de nuevo, tanto puramente intelectuales como de carácter práctico. La cuestión que podemos plantearnos ahora es la de si se puede establecer alguna relación entre ambos ámbitos, el lingüístico y el del tipo de actividad socio-cultural.

3.1. Sabemos que existen relaciones entre los recursos gramaticales utilizados por las lenguas y la búsqueda de facilidad de procesamiento que en gran medida se corresponde con lo que en un ámbito funcional, no cognitivo, llamamos 'economía del lenguaje', aunque también sabemos que estos recursos económicos están supeditados a la naturaleza tipológica de las lenguas. En efecto, las relaciones entre la forma de los enunciados - por ejemplo, en las preferencias en lo concerniente al orden de palabras- y las facilidades de procesamiento han sido profusamente estudiadas (Bock, 1982; Bock, Warren, 1985; Wasow, 1997; Arnold et al., 2000; Hawkins, 2004; MacDonald, 2013, entre otros muchos) y no voy a entrar en el tema que nos aleja de lo que aquí interesa. De los estudios hechos se desprende, asimismo, que la 'facilidad de procesamiento' no es un término absoluto e independiente, sino que está ligado a otras tendencias de las lenguas. Por ejemplo, la tendencia a anteponer los elementos cortos frente a los largos, constatable en lenguas como el inglés y, en general, las de orden VO, no opera del mismo modo en las lenguas OV como japonés o coreano (Yamashita, Chang, 2001), o vasco (Ros et al., 2015) ${ }^{10}$.

10 De acuerdo con Ros et al. (2015, 8-10): "in VO languages the shortbefore long word order might arise not only from the preference to place shorter elements earlier in the sentence, but also from the preference to avoid complex constituents in the middle of the sentence, which are more costly to process [...] For instance, (5a) is harder to process than (5b) below, because the relative clause embedded within the direct object increases the distance between the verb and the indirect object, making the integration of the indirect object harder. Therefore, speakers may prepose the indirect object to reduce the processing cost. In strictly OV languages, however, these two constraints pull in different directions: a short-before- 
Podemos considerar, por tanto, que aunque la búsqueda de la economía, que conlleva como parte de la misma la 'facilidad de procesamiento', es una característica de las lenguas -y de los hablantes-, los modos de conseguirla no son uniformes y están supeditados a rasgos estructurales y tendencias de las lenguas.

3.2. Se han visto, sin embargo, algunas posibles correspondencias entre hábitos intelectuales de los hablantes y rasgos asociados indirectamente a las lenguas, en concreto, los sistemas de escritura. Así, se han tratado de encontrar correspondencias entre sistema de escritura y formas de encarar las operaciones mentales y, en última instancia, de dar forma a la aportación colectiva. Torres i Graell (1983) en su excelente libro sobre la escritura japonesa, llama la atención sobre el hecho de que los pueblos que adoptan la escritura china, independientemente de la naturaleza tipológica de sus lenguas, crean un hábito de lectura 'analítica', pues en ellos leer significa ver de golpe una serie dibujos cuya comprensión textual exige ir separándolos, esto es, analizándolos adecuadamente. Un ejemplo del uso de la escritura ideográfica en japonés puede ilustrarnos este proceso de 'análisis' o separación ${ }^{11}$ : La secuencia (1) del japonés:

\section{(1) 軽い軽食を食べる}

long linearisation of constituents places short elements earlier in the sentence, but it also creates a center-embedded structure. In these cases, speakers should seek to avoid center-embedding by shifting longer constituents to a sentence initial position, leading to a long-before-short ordering. [...]

(5a) I [VP gave [NP the book she had been searching for since last Christmas] [PP to Bill]]

(5b) I [VP gave [PP to Bill] [NP the book she had been searching for since last Christmas]]".

11 Agradezco al profesor Nakai Kuniyoshi, de la Universidad Ritsumeikan de Kioto (Japón), el haberme facilitado ejemplos como el que utilizo y, en general, haberme solucionado todas las dudas sobre la lengua japonesa que le he ido presentando. 
Tendría la siguiente descomposición:

$\begin{array}{llll}\text { 軽い } & \text { 軽食 } & \text { を } & \text { 食べ12 } \\ \text { karu-i } & \text { KEI-SHOKU } & \text { o } & \text { ta- be - ru } \\ \text { ligero } & \text { ligera comida } & \text { ACUS. } & \text { comer } \\ \text { (= comer ligero comida ligera) } & & \end{array}$

Los signos se repiten, pero hasta que no se individualizan en cada contexto no se pueden identificar las palabras concretas que conforman el texto. Así, el mismo signo 軽, con el significado 'ligero' puede ser:

軽 karu (i) Adjetivo (con valor adverbial). Palabra aislada (con morfema ' $i$ ' (い) de presente afirmativo) pronunciada 'a lo japonés' (kun)

軽 KEI Adjetivo. Parte de un compuesto, pronunciado 'a lo chino' (on)

Del mismo modo, 食 será en un caso un sustantivo y en otro un verbo:

食 SHokU Sustantivo parte de compuesto. Pronunciación ON

食 $t a$ (be-ru) Verbo. Palabra aislada (con morfemas 'be' + 'ru' (ベ+る) de presente). Pronunciación kun.

12 Los elementos gráficos い, を, べ y る no corresponden a ideogramas chinos, sino a un silabario propiamente japonés con el que expresan morfemas de carácter aglutinante, tanto verbales como casuales. Son ajenos a la escritura de la lengua china y específicos de la lengua japonesa que, de este modo, utiliza al tiempo un sistema ideográfico (igual que el chino), y un sistema fonográfico (silabario) con el que expresa morfemas y, a veces, también palabras enteras. Las palabras japonesas tienen siempre dos (o más) lecturas, una propiamente japonesa y otra 'china', que se suele utilizar cuando las palabras forman compuestos. En la transcripción que hago utilizo la letra cursiva para la transcripción de las palabras que tienen una pronunciación 'japonesa' y versal para las que tienen una pronunciación 'china'. 
Esto es, el proceso que cualquier lector de japonés debe seguir para decodificar correctamente esta secuencia sería:

1. Se ve de un golpe la secuencia (軽い軽食を食べる).

2. Se separan unidades significativas (palabras) (軽(い) + 軽 食 $+($ を + 食(べる)) en un proceso inicial de análisis.

3. Se recupera el sentido global en un proceso final de síntesis.

En la escritura alfabética (fonográfica, en general), en cambio, tenemos una serie de signos fonéticos sin sentido por sí mismos, y para que podamos entenderlos tenemos que juntarlos (un proceso inicial de síntesis) para, así, poder leer cada palabra. Por ello, el proceso de lectura japonesa antes descrito contrasta con el de la decodificación de un texto en escritura alfabética, silábica o, en general, fonográfica. Si queremos leer y entender algo como

(2) Cuentan de un sabio que un día...

tendremos que dar los siguientes pasos:

1. Ver las letras que corresponden a unidades fónicas sin significado: $c-u-e-n-t-a-n \ldots$

2. Juntarlas para que formen un todo identificable (sílabas): cuen-tan-de...

3. Volver a juntar esas partes en distintos todos para que formen unidades significativas (cada palabra con sus morfemas): cuentan de...

4. Volver a unir, ahora en un proceso igual al del sistema anterior, para dotar de sentido global a las distintas unidades reconocidas por síntesis.

Este proceso de síntesis normalmente se produce de modo jerarquizado, pues las palabras léxicas y gráficas suelen contener composiciones y en nuestra decodificación, más que una síntesis, se producen varias síntesis jerarquizadas. Naturalmente a partir de un momento del aprendizaje de la lectura muy temprano este proceso se ha automatizado en el lector de tal manera que tene- 
mos la impresión de que los pasos ilustrados en (3-4) realmente no los damos, pues la imagen visual de la palabra la captamos 'de un golpe’ a fuerza de costumbre. Pero no deja de ser una ilusión. Cuando nos enfrentamos con una palabra perteneciente a una lengua que no dominamos lo suficiente como para tener automatizada su captación visual global, el proceso de unión se muestra claramente. Palabras como las incluidas en (3), para quienes no dominen el sánscrito, exigen un esfuerzo de 'síntesis' como el ilustrado arriba para captarlas adecuadamente:

(3) tacchailasikhare Ghantakarno nama raksasah prativasatiti janapavadah sada sruyate ${ }^{13}$.

No es casual -dice Torres i Graell- que en japonés 'entender' se diga wakaru (分か), donde la raíz wa (分) significa 'separar', mientras que en latín se utilizara el término comprehendere (cum + prehendere), inicialmente 'asir algo', del que deriva el adjetivo comprehensibilis con el significado de 'asir algo intelectualmente' $y$, posteriormente, simplemente 'entender'. Estas distintas 'técnicas de lectura' hacen, según este autor, que "el oriental se ha convertido en un hombre de mentalidad inductiva en vez de deductiva como lo es la del occidental" $(1983,20)$, llegando a establecer una relación entre estos hábitos de técnica lectora y el modo de afrontar las operaciones intelectuales de los japoneses. En sus palabras:

El predominio del análisis sobre la síntesis ha influido indudablemente en la forma de desarrollo tecnológico del Japón. Se puede constatar que su investigación básica en los diferentes campos de la ciencia y de la técnica no ha destacado en comparación con otros países. La capacidad del japonés de obtener resultados concretos a partir de premisas abstractas es inferior a la del occidental. Sin embargo su facilidad para analizar y pro-

13 Secuencia del Hitopadesha que significa Se oye siempre un rumor entre la gente: un demonio llamado Gantha-Karno (el de la campana en la oreja) habita en la cumbre de esta montaña. Para simplificar utilizo la transcripción en caracteres latinos, y no su escritura devanagari original. 
fundizar en un objetivo determinado es muy superior, permitiéndole mejorar y conseguir un alto grado de perfeccionamiento que ha supuesto la base del rápido y fantástico despegue tecnológico del Japón moderno (Torres i Graell, 1983: 20).

Es curioso comprobar la similitud de las afirmaciones de Torres i Grael con las de Schlegel más de siglo y medio antes, según quien el espíritu antiguo (sintético) era más apropiado para las grandes creaciones, mientras que el moderno (analítico) lo era para su perfeccionamiento. En sus palabras:

Je pense qu'en comparant le génie de l'antiquité avec l'esprit des temps modernes, on observera une opposition semblable à celle qui existe entre les langues. Les grandes synthèses créatrices sont dues à la plus haute antiquité; l'analyse perfectionnée était réservée aux temps modernes (Schlegel, 1818: 27-28).

3.3. La cuestión que planteo aquí, sin embargo, no es la de la asociación de rasgos tipológicos de la lenguas y su forma de elegir la forma de sus enunciados, ni la posible relación de un sistema lingüístico 'sustitutorio', como el escritural, con hábitos mentales, sino la posible relación entre los rasgos tipológicos, preferentemente las tendencias sintácticas, y la forma de actuar de sus hablantes. Esto es, la forma de afrontar la comunicación y la forma de afrontar la vida y, más concretamente, los rasgos sintácticos del latín que apuntan a su carácter sintético y el tipo de legado, que apunta a intereses y formas de actuación muy concretas y prácticas.

Debo decir que no conozco estudios que traten la relación entre la estructura de las lenguas, como tal, y su facilidad de procesamiento. Por ejemplo, no conozco trabajos que hayan afrontado la pérdida de los rasgos morfológicos del inglés a favor de soluciones más 'analíticas' como el resultado de una busca de comodidad de procesamiento y de emisión-recepción de la información. O la posible relación de la concisión sintética de las secuencias del inglés (phrasal verbs, construcciones resultativas, abundancia de composición...) y la búsqueda de la 'comodidad comunicativa', quizá porque, como he señalado anteriormente, esa comodidad no es independiente de otros 
factores estructurales. De otro modo, todas las lenguas adoptarían soluciones similares en búsqueda de ese universal comunicativo que es la economía del lenguaje. Por ello, el establecimiento de relaciones entre el legado romano y la lengua latina que abordaré brevemente a continuación no se hará con la pretensión de afirmar relaciones de causa-efecto o implicativas en general. Tan solo pretende constatar concomitancias entre, por una parte (i) planteamientos políticos y socio-culturales de carácter práctico llevados a cabo por hablantes de lenguas sintácticamente sintéticas y por otra (ii) planteamientos diferentes de hablantes de lenguas sintácticamente analíticas, centrándome en el contraste entre el mundo romano y el mundo posterior a la caída del imperio, sin otras pretensiones.

\section{4.}

\section{La lengua latina y el legado latino}

4.1. Desde el punto de vista tipológico, el latín puede caracterizarse como una lengua todavía sintética morfológicamente. Digo todavía porque desde lo que sabemos del proto-indoeuropeo, la lengua latina había sufrido ya un proceso de adopción de rasgos analíticos que se iría incrementando en sus herederas. Para simplificar, podemos condensar en un cuadro los rasgos de carácter sintético del protoindoeuropeo que en latín se transformaron en analíticos, esto es, el avance de la tendencia analítica del latín con respecto al proto-indoeuropeo.

Cuadro 3. Rasgos contrastivos protoindoeuropeo - Latín

\begin{tabular}{|l|l|l|}
\hline & Protoindoeuropeo & Latín \\
\hline 1. & Sistema casual rico & Sistema casual se simplifica y se empobrece \\
\hline 2. & Ausencia de partículas: & - Aparecen las preposiciones con acusativo \\
& - preposiciones & y ablativo. En este último caso con enorme \\
& - artículos & frecuencia \\
& Tendencia a la conjunción & - Prolijo uso de partículas \\
enclítica & \\
\hline
\end{tabular}




\begin{tabular}{|c|c|c|}
\hline & Protoindoeuropeo & Latín \\
\hline 3. & $\begin{array}{l}\text { Sistema verbal complejo: } \\
\text { - tiempos verbales } \\
\text { abundantes en formas simples } \\
\text { - hasta cinco modos verbales } \\
\text { diferenciados }\end{array}$ & $\begin{array}{l}\text { - En latín tardío, prolijo uso de demostrativos } \\
\text { (inicialmente en contextos nominativos), } \\
\text { antesala del artículo románico } \\
\text { - Se incrementan las conjunciones exentas } \\
\text { y desaparecen las enclíticas (solo queda -que) } \\
\text { - Reducción de las formas temporales, } \\
\text { y comienzo del uso de las formas } \\
\text { perifrásticas } \\
\text { - Reducción de los modos verbales a dos: no } \\
\text { marcado (indicativo) y marcado (subjuntivo) }\end{array}$ \\
\hline 4. & $\begin{array}{l}\text { Tendencia a la composición } \\
\text { léxica } \\
\text { A veces con formación de } \\
\text { unidades léxicas equivalentes } \\
\text { a unidades predicativas }\end{array}$ & $\begin{array}{l}\text { - Progresivo incremento de la derivación en } \\
\text { detrimento de la composición } \\
\text { - En el caso de la prefijación, inventario de } \\
\text { prefijos a menudo coincidente con el de las } \\
\text { preposiciones y de algunos de los iniciales } \\
\text { adverbios }\end{array}$ \\
\hline 5. & $\begin{array}{l}\text { Construcciones sintácticas } \\
\text { muy compactas y a menudo } \\
\text { rígidas: } \\
\text { - construcciones absolutas } \\
\quad \text { (solo) con participio } \\
\text { - frases nominales puras } \\
\quad(\mathrm{FNP})^{*}\end{array}$ & $\begin{array}{l}\text { - Comienzo de la liberalización de las } \\
\text { construcciones sintácticas } \\
\text { - Construcciones absolutas con varios } \\
\text { componentes } \\
\text { - Menor uso de la FNP, relegada a contextos } \\
\text { específicos y sustituida por construcciones } \\
\text { copulativas }\end{array}$ \\
\hline
\end{tabular}

* La FNP es una construcción bimembre que consta de un elemento presentativo (temático) y un atributo. En español un ejemplo de FNP sería: El ladrón, atrapado.

El cuadro 3 caracteriza el latín como una lengua que camina hacia recursos analíticos, pero si la comparamos con sus derivaciones posteriores, aparece como una lengua de una idiosincrasia todavía fundamentalmente sintética. De nuevo ahorramos espacio mediante un cuadro que compara de un modo esquemático los recursos sintéticos del latín en relación con los más analíticos de las lenguas románicas y, muy específicamente, del español. El cuadro 4 muestra los rasgos de la tendencia analítica del español, en su contexto románico, en relación con los del latín, tanto en su 
vertiente morfológica como en la sintáctica. En este momento no puedo extenderme en estos extremos, de ahí que delegue en estos cuadros los rasgos que merecen ser destacados:

Cuadro 4. Rasgos del contraste sintético (latín) / analítico (español)

\begin{tabular}{|c|c|c|}
\hline & Latín & Español \\
\hline \multirow[b]{2}{*}{$\begin{array}{l}\text { Creación } \\
\text { de nuevas } \\
\text { unidades }\end{array}$} & $\begin{array}{l}\text { Ausencia de artículo } \\
\text { o determinantes átonos } \\
\text { y uso prolijo de demos- } \\
\text { trativos en latín tardío }\end{array}$ & $\begin{array}{l}\text { 1. Uso complejo del ARTÍCULO en } \\
\text { combinación con su ausencia y con otros } \\
\text { determinantes }\end{array}$ \\
\hline & $\begin{array}{l}\text { Ausencia total de clíticos } \\
\text { Donavisti librum puellae? } \\
\text { Donavi. } \\
\text { Puellam hodie vidimus }\end{array}$ & $\begin{array}{l}\text { 2. Inventario reducido (frente a francés } \\
\text { o italiano) pero uso muy prolijo de los } \\
\text { CLÍTICOS (tematizaciones, avance } \\
\text { catafórico) } \\
\text { — ¿Has dado el libro a la niña? } \\
\text {-Sí, sE Lo he dado } \\
\text { A la niña LA hemos visto hoy }\end{array}$ \\
\hline \multirow{4}{*}{$\begin{array}{l}\text { Incremento } \\
\text { de uso } \\
\text { de unidades } \\
\text { con casos de } \\
\text { reanálisis }\end{array}$} & $\begin{array}{l}\text { Uso muy incipiente de } \\
\text { ciertas perífrasis con } \\
\text { essere y habere }\end{array}$ & $\begin{array}{l}\text { 3. Las PERÍFRASIS VERBALES abarcan } \\
\text { la expresión de una gran gama de conte- } \\
\text { nidos modales y aspectuales }\end{array}$ \\
\hline & $\begin{array}{l}\text { Voz medio-pasiva mixta } \\
\text { (sintética y analítica) }\end{array}$ & $\begin{array}{l}\text { 4. Uso extremadamente productivo de la } \\
\text { VOZ MEDIA con la construcción con SE } \\
\text { Distribución complementaria con el } \\
\text { dativo de interés }\end{array}$ \\
\hline & $\begin{array}{l}\text { Preposiciones especi- } \\
\text { ficadoras del valor del } \\
\text { acusativo y del ablativo }\end{array}$ & $\begin{array}{l}\text { 5. Las PREPOSICIONES pierden to- } \\
\text { talmente su sistematicidad y la mayor } \\
\text { parte de las (pocas) heredadas del latín } \\
\text { se convierten en marcas gramaticales } \\
\text { desemantizadas }\end{array}$ \\
\hline & $\begin{array}{l}\text { Construcciones sinté- } \\
\text { ticas } \\
\text { (Mali et bona, Intelligen- } \\
\text { tibus, pauca, Fortissimus } \\
\text { quisque, Triste lupus } \\
\text { stabulis ...) frecuentes en } \\
\text { la lengua literaria }\end{array}$ & $\begin{array}{l}\text { 6. Construcciones directas arrinconadas } \\
\text { a distintas manifestaciones fraseológicas } \\
\text { (Casa con dos puertas, mala de guardar...) } \\
\text { Poco uso incluso para titulares de pe- } \\
\text { riódicos u otros contextos que requieren } \\
\text { lenguaje sintético }\end{array}$ \\
\hline
\end{tabular}


El cuadro retrata el latín como una lengua mucho más austera en recursos expresivos explícitos que sus descendientes románicas. Sin llegar a la condensación sintáctica del inglés, conserva construcciones muy sintéticas, y morfológicamente mantiene todavía gran parte de la rentabilidad flexiva indoeuropea, parte de la cual se perderá en la lenguas románicas, aunque conserven lo esencial de la flexión verbal y parte de la nominal.

4.2. Si ahora observamos, siquiera superficialmente, el tipo de legado que conservamos de Roma, esto es de la cultura que habló y transmitió el latín, observamos que dominan los planteamientos globales con fines prácticos, lo que en el cuadro 2 quedaba encuadrado como I.2, al lado de legados como el de la Gran Bretaña colonial. Roma fue un imperio que buscó, ante todo, consolidar su poder asimilando los pueblos que conquistaba y, para ello, les dotaba de todo el andamiaje necesario para la integración. Su espíritu práctico se manifiesta, no solo en el despliegue de instituciones, obras, infraestructuras y trazados que dejó a lo largo de su imperio, sino en la propia conciencia que sobre ello muestran autores como Cicerón. En el libro II de su Diálogo Sobre la República hay un conocido pasaje en el que Escipión alude a Catón el Viejo, quien solía decir que:

[...] la constitución de nuestra sociedad aventajaba a las de las otras ciudades, porque en ellas habían sido hombres aislados y cada uno de ellos había constituido la República con sus propias leyes e instituciones, como Minos la de Creta, Licurgo la de Lacedemonia, y la de Atenas había cambiado muchas veces por obra de Teseo, de Dracón, de Solón, de Clístenes y de otros muchos; finalmente la había sustentado un varón sabio, Demetrio Falereo, cuando ya se encontraba extenuada y moribunda. Nuestra constitución, en cambio, no era fruto de uno solo, sino de muchos; ni se consolidó en una generación, sino que fue mudando a lo largo de los siglos y de las generaciones (Cicerón, Republica, II, 49).

Pero, además de ponderar el hecho de que la constitución de Roma haya sido hecha de forma colectiva, solidaria y, por ello, en gran medida con un carácter 'práctico', abundan los testimonios en los que los romanos se enorgullecen de la utilidad de 
sus construcciones, y no solo de su belleza. El abastecimiento de aguas, por poner un ejemplo significativo (pero en absoluto único) despertó todo tipo de admiraciones entre propios y extraños:

[...] Al menos yo, entre las tres construcciones más magníficas de Roma, por las que principalmente se muestra la grandeza de su poder, coloco los acueductos, los pavimentos en los caminos y las obras de las cloacas, y esta opinión no se refiere solo a la utilidad de la construcción, sino a la magnitud de los gastos (Dionisio de Halicarnaso, III, 67, 5).

Otros testimonios a menudo citados por los historiadores apuntan en la misma dirección. Así, Frontinus, curator de agua con Nerva, (siglo I) señala:

A los beneficios tan numerosos y tan necesarios de tantos acueductos van pues a comparar las pirámides, que no sirven obviamente para nada o también las obras de los Griegos, inútiles pero celebradas por todas partes (Frontinus, S. J. (s. I): De aquaeductu, XVI, 1, apud Litaudon, 2002:3).

Igualmente, Plinio el Viejo siente gran admiración por cuestiones tan complejas y tan prácticas como las conducciones acuíferas:

Pero si alguien calculara minuciosamente la cantidad de agua de los suministros públicos, baños, depósitos, casas, zanjas, jardines y villas suburbanas; $y$ por la distancia que deben atravesar, los arcos construidos, las montañas perforadas, los valles nivelados; tendremos que confesar que no ha habido nada tan maravilloso en todo el mundo (Plinio, Naturalis Historia XXXVI, 15, apud De la Peña Olivas, 2010: 249).

Los testimonios de admiración sobre obras hechas con sentido práctico se pueden extender, pero aquí nos interesa fundamentalmente destacar que ese espíritu práctico que se desprende del legado de Roma era algo conscientemente buscado y de lo cual sus historiadores se mostraban orgullosos. 
4.3. ¿Existe realmente algún tipo de relación entre este espíritu práctico de Roma y el carácter sintácticamente sintético de su lengua? Es difícil asegurar nada al respecto, pero es un hecho que el carácter práctico y resolutivo del legado romano dio paso en el mundo que le siguió a un espíritu muy distinto, a la par que un tipo de lenguas mucho menos sintéticas, un espíritu fundamentalmente exegético, con actividades más dedicadas a cuidar, interpretar y desarrollar logros heredados que a innovar y pensar en soluciones a problemas de organización y convivencia, lo cual invita a establecer correspondencias entre tipos de lenguas y tipos de actividad. Podemos decir que la actividad medieval del mundo románico cuidó más aspectos de desarrollo y perfeccionamiento de modelos previos que verdaderas innovaciones en el campo de la cultura y las artes. Incluso en el campo de las lenguas, las primeras gramáticas de las lenguas vulgares, con Nebrija (1492) a la cabeza, siguieron un modelo latino a rajatabla, sin nuevos planteamientos. Es cierto que los romanos habían tomado el armazón gramatical de los griegos, pero a partir de él construyeron gramáticas propiamente del latín. Las nuevas gramáticas de las lenguas romances que se hicieron a partir de finales del siglo XV tardaron bastante en desembarazarse de descripciones latinas que no tenían que ver con la lengua que estaban describiendo ${ }^{14}$.

Ahora bien, de nuevo ¿podemos considerar que el carácter global y práctico del legado romano, frente al disperso y caduco en el tiempo de gran parte del quehacer medieval está en relación con el carácter (más) sintético del latín frente al claramente analítico de las lenguas romances?

La respuesta inicial a esta pregunta es que 'difícilmente' se pueden asociar, pues tanto las lenguas como las culturas se modifican sin que el ritmo de las modificaciones de unas y otras sea

14 Según Nebrija, "Nombre es una delas diez partes de la oracion que se declina por casos, sin tiempos; i significa cuerpo o cosa" (Nebrija, 1492, III, II, vol. 2 pág. 58. Cursiva mía). Como se ve, pese a lo novedoso de su gramática, pues fue la primera de una lengua vulgar en Europa, Nebrija calca los modelos latinos y atribuye al nombre castellano unos casos que habían dejado de existir en el habla de la gente hacía más de siete siglos. 
el mismo necesariamente. Está claro que lenguas como el inglés o el español son habladas por pueblos muy distintos y no responden a un patrón de comportamiento único.

Una vez hecha la primera reflexión, sin embargo, hay que reconocer que las variadas culturas que han adoptado el inglés o el español son producto de la colonización o de la emigración, con el consiguiente desplazamiento de los hablantes a un nuevo medio con nuevos entornos, contactos y necesidades. Ello conlleva la pérdida de gran parte de la cultura original, a veces con el resultado de producirse un rechazo de estos emigrantes a su pueblo de procedencia, aunque conserven y usen la lengua importada (lo que ocurre en gran parte de Hispanoamérica con los sentimientos antiespañoles de descendientes de españoles). La dispersión cultural e idiosincrática, por tanto, viene después. Pero esta dispersión posterior no es significativa. Las características tipológicas del español de hoy día se forjaron en la Península Ibérica, partiendo de la zona nororiental, desde finales de la Alta Edad Media en adelante, y los rasgos del español moderno vienen ya del siglo XVI, de mismo modo que los rasgos del inglés actual se forjaron en Inglaterra con el inglés moderno temprano, a partir del siglo XV. Y en este sentido, el contraste entre las culturas inglesa e hispánica y, al tiempo entre sus lenguas, es notorio.

La España que forja el idioma español no destaca como la sociedad británica en cuanto a soluciones prácticas. Es cierto que la leyenda negra española ha contribuido a minimizar los planteamientos en cuanto a infraestructuras y legislaciones, y que se ha hecho mucho más hincapié en la rentabilización de las riquezas que el nuevo mundo les ofrecía que en su legado positivo, pero también es cierto que incluso en la labor práctica de publicitar sus logros España ha sido muy poco eficiente, perdiendo claramente la batalla propagandística con sus enemigos protestantes ${ }^{15}$. La

15 Un interesantísimo y magníficamente documentado texto sobre la leyenda negra española y la minimización de sus logros en América se encuentra en Roca Barea (2016, Parte II cap. 7), obra, por cierto, contestada furibundamente por Villacañas (2019). 
visión que se ha generalizado del periodo tras el descubrimiento de América es que España no estuvo bien gobernada y a menudo se perdían esfuerzos, talentos y dinero por falta de 'sentido de estado' o 'visión histórica', esto es, por no saber establecer adecuadamente las prioridades y perderse en cuestiones secundarias que no llevaban a nada (o, peor, que llevaban a la ruina) ${ }^{16}$. Tampoco el pensamiento español en esta época creó modelos que trascendieran, y en las historias de la filosofía o del pensamiento mundial o europeo España no destaca precisamente como país fuente de inspiración, estando muy por debajo, no solo de Alemania o Inglaterra, sino también de Italia o Francia. Solo en la creación literaria puede competir, pero solo a partir del siglo XVI y en gran medida, por el empuje y difusión internacional de la figura de Cervantes y su obra Don Quijote. En suma, no ha habido en la España desde el medievo una visión global que le llevara a creaciones teóricas o a soluciones prácticas perdurables, sino atomización de logros inconexos ${ }^{17}$.

El contraste de la historia española con lo que ocurrió en Inglaterra cuando comenzó a fraguarse el más tardío imperio británico, es llamativo. Para empezar, Inglaterra es la creadora del empirismo como teoría filosófica, y Francis Bacon (1561-1626) pasa por ser el creador de la mecánica, esto es, la ciencia puesta al servicio de fines prácticos. Como potencia colonial, Inglaterra, sin llegar a los extremos excelsos de Roma, planificó unas infraes-

16 Es altamente recomendable la lectura de Eslava Galán (1995) para tener una visión clara (y amena) de los desastres a los que llevó la falta de espíritu 'sintético' de los gobernantes españoles a lo largo de los siglos.

17 Es proverbial la aparición en España de figuras que destacan sorprendentemente en campos en los que no hay una planificación para su fomento. Manuel Santana en tenis, en los años sesenta del siglo XX, o Severiano Ballesteros en golf posteriormente, fueron claros ejemplos de figuras tan meritorias como sorprendentes. O, en el terreno musical, grandes intérpretes y cantantes de ópera han surgido cuando la educación musical en España estaba -y está- lejos de tener la consistencia y planificación de la mayoría de los países del entorno, por citar casos bien conocidos por todos. 
tructuras a largo plazo. Tan a largo plazo que hoy día, setenta años después de la independencia de la India, sus ferrocarriles circulan por el trazado hecho por los británicos. El espíritu 'práctico' de los británicos es proverbial, tanto para lo bueno como para lo no tan bueno.

4.4. No deja de ser curioso que las similitudes de visión y actuación de Roma y el imperio británico, salvando distancias temporales y culturales, esté acompañado de lenguas de carácter sintácticamente sintético, mientras que la historia de España, con una trayectoria muy distinta, se ha desarrollado con una lengua muy analítica, donde abundan las partículas a menudo superfluas desde el punto de vista gramatical o semántico referencial, pero implantadas en el uso. Es como si el espíritu práctico mostrado por romanos y británicos a la hora de afrontar las tareas de la vida quedara reflejado en su lengua, con la supresión de recursos sintácticos innecesarios ${ }^{18}$, la compresión de la información en unidades compactas y, en general, la eliminación de elementos gramaticales superfluos, mientras que España, perdida en su historia en episodios evitables y con una idiosincrasia lejana a ese espíritu práctico y riguroso de los romanos, y bien plasmada en la literatura, fue desarrollando una lengua de tendencias sintácticas marcadamente analíticas, probablemente la más analítica de entre las románicas, llena de usos del artículo incomprensibles para los extranjeros y difíciles de explicar para los profesores de ELE, de clíticos usados sin el menor sentido práctico, de perífrasis que sustituyen la pérdida de formas verbales simples, de preposiciones creadas en aluvión, de estructuras sintácticas que no omiten ningún tipo de marca de relación y, desde el punto de vista paradigmático, caracterizada por la posesión de subsistemas relevantes en la expresión sintáctica (preposiciones, conjunciones, relativos,

18 Insisto en el carácter sintético de los recursos sintácticos porque en sus unidades morfológicas el inglés es puesto como ejemplo de lengua analítica, por su pérdida de flexión y el consiguiente uso constante de pronombres y verbos auxiliares. 
partículas completivas) de uso obligado allí donde aparecen, pero en algunos casos (preposiciones, conjunciones) muy poco sistemáticos, casi a nárquicos.

Es cierto que se pueden poner ejemplos de culturas en las que la lengua y los rasgos de la comunidad no se corresponden en el sentido visto arriba, pero es que en las características estructurales y de uso de una lengua entran muchos factores que hacen que no haya lenguas tipológicamente puras, como tampoco existen culturas unidireccionales, aunque a la hora de juzgar su legado nos quedemos con una abstracción (la misma que crea estereotipos de los países y de las gentes). Pero las coincidencias existen ${ }^{19}$. Pensar que pueda haber una relación más allá de la mera coincidencia no es descabellado, al menos a un nivel tipológico general.

\section{5. \\ Reflexión final}

Como apunte telegráfico final podemos decir que la mentalidad de un individuo o de un pueblo es algo global, que de algún modo impregna la lengua, de modo que la forma en que se usa refleja en alguna medida esta mentalidad. Naturalmente no hay una implicación necesaria entre formas de ser y formas de hablar. En la estructura y usos de una lengua influyen multitud de factores, su origen, sus contactos, las necesidades comunicativas que se adquieren o se pierden. Ello hace que no haya ni lenguas ni culturas tipológicamente puras, pero del mismo modo que ninguna lengua refleja fielmente la idiosincrasia de sus hablantes, ninguna lengua puede sustraerse a la influencia de las ne-

19 En esta misma línea podría buscarse una relación entre el hecho de que el portugués del Brasil adquiera rasgos más analíticos que el de Portugal, por ejemplo, en el uso de clíticos de voz media o en perífrasis verbales (Sousa Pereira, 2007), y la misma idiosincrasia de los pueblos brasileño y portugués. 
cesidades y costumbres de las comunidades que la hablan. De hecho esa influencia suele ser el primer paso del cambio lingüístico, por lo que no es extraño que se encuentren correspondencias entre tendencias lingüísticas y concepciones de la realidad, intereses y objetivos de sus hablantes. Por lo que podemos ver, la lengua latina es buena muestra de las formas expresivas que casan perfectamente con una cultura de gente práctica, que busca más planteamientos globales y útiles que ornamentos superfluos y que, tanto a la hora de actuar como a la hora de hablar tiende a ir "al grano". Que ese hermanamiento sea puramente casual o motivado ya es materia, al menos, merecedora de una atención más profunda y documentada.

\section{Bibliografía}

Arnold, J. E., Losongco, A., Wasow, T., Ginstrom, R. (2000): “Heaviness vs. newness: The effects of structural complexity and discourse status on constituent ordering". Language 76 (1): 28-55.

Bock, J. K. (1982): “Toward a cognitive psychology of syntax: Information processing contributions to sentence formulation”. Psychological Review 89 (1): $1-47$.

Bock, J. K., Warren, R. K. (1985): "Conceptual accessibility and syntactic structure in sentence formulation". Cognition 21 (1): 47-67.

Bussmann, H. (1996): Routledge Dictionary of Language and Linguistics. Translated and edited by Gregory Trauth and Kerstin Kazzazi, London/ New York: Routledge.

Conze, E. (1978): El budismo. Su esencia y su desarrollo. México: Fondo de Cultura Económica.

De la Peña Olivas, J. M. (2010): "Sistemas romanos de abastecimiento de aguas”. En: Las técnicas y las construcciones en la ingeniería romana. Córdoba (Congreso de las obras públicas romanas), 249-282.

Dionisio de Halicarnaso (1984): Historia antigua de Roma. Traducción española y notas de E. Jiménez y E. Sánchez. Madrid: Gredos.

Dixon, R. M. W. (1994): Ergativity. Australian languages: their nature and development. Cambridge: Cambridge University Press. (Cambridge Studies in Linguistics, 69). 
Eslava Galán, J. (1995): Historia de España contada para escépticos. Barcelona: Planeta.

Greenberg, J. H. (1960): "A Quantitative Approach to the Morphological Typology of Language”. International Journal of American Linguistics 26: 178-194.

Greenberg, J. H. (1963): "Some Universals of Grammar with Particular Reference to the Order of Meaningful Elements". En: J. H. Greenberg, (ed.). Universals of Language. London: MIT Press, 73-113.

Hawkins, J. A. (2004): Efficiency and complexity in grammars. Oxford: Oxford University Press.

Her, W. I. (2002): La formación de palabras en chino y en español (tesis doctoral). Madrid: Universidad Complutense.

Jiménez Juliá, T. (2017a): "Rasgos del español como lengua analítica”. Studia Romanistica 17 (1): 99-116.

Jiménez Juliá, T. (2017b). "Notas sobre lenguas analíticas y sintéticas desde una óptica sintáctica”. En: J. L. Aliaga (ed.), En el centenario del Cours (1916) de Saussure. Pervivencia de la obra y nuevos retos de la lingüística. Zaragoza: Institución Fernando el Católico (en prensa).

Jiménez Juliá, T., Doval Reixa I. (2014). "Notes on Germanic direct constructions. A contrastive approach with Spanish”. En: I. de la Cruz Cabanillas, C. Tejedor Martínez (eds.), Linguistic Insights. Studies on Languages. Alcalá de Henares: Universidad de Alcalá, 323-335.

Lévi-Strauss, C. (1958): Anthropologie structurale. Paris: Plon.

Litaudon, J. C. (2002): "Acqua Lugduniensis. El acueducto romano del Gier". Traducción de I. Moreno Gallo, http://traiavans.net/ [15.08.2017].

Malinowski, B. (1923): "The Problem of Meaning in Primitive Languages". Supplement I de C. K.. Ogden, I. A. Richards, The Meaning of Meaning. London: Kegan Paul, 296-336.

MacDonald, M. C. (2013): "How language production shapes language from and comprehension". Frontiers in Psychology 4: 1-16.

Mendívil Giró, J. L. (2003): “Construcciones resultativas y gramática universal”. Revista Española de Lingüística 33 (1): 1-28.

Moreno Cabrera, J. C. (2003): "Síntesis y análisis en las lenguas. Crítica de la tipología morfológica clásica y de algunas de sus aplicaciones sincrónicas y diacrónicas". ELUA 17: 465-504.

Nebrija, A. (1492): Gramática castellana. Madrid: Edición Junta del Centenario (2 vol.), 1946. 
Pabón, J.M. (1967): Diccionario manual Griego-Español Vox. Barcelona: Bibliograf.

Radcliffe-Brown, A. R. (1952): Structure and function in primitive society. London: Cohen and West.

Real Academia Española (1726): Diccionario de autoridades. Edición Facsímil. Madrid: Gredos, 1963.

Roca Barea, M. E. (2016): Imperiofobia y leyenda negra. Madrid: Siruela.

Ros, I. (2015): "Aiming at shorter dependencies: the role of agreement in morphology, Language, Cognition and Neuroscience 30. Issue 9: Laboratory in the field: Advances in cross-linguistic psycholinguistics, http://dx.doi. org/10.1080/23273798.2014.994009 [15.08.2017].

Schlegel, A. (1818): Observations sur la langue et la littérature provençales. Paris: Librairie grecque-latine-allemande.

Siegel, J., Szmrecsanji, B., Kortmann, B. (2014): “Measuring analyticity and syntheticity in creoles". Journal of Pidgin and Creole Languages 29 (1): 49-85.

Sorbet, P. (2017). “Los mecanismos de creación léxica en español y en polaco". Studia Romanistica 17 (1): 129-140.

Sousa Pereira, S. de (2007): Estudio contrastivo del régimen verbal en el portugués de Brasil y el español peninsular. Tesis Doctoral inédita. Universidad de Santiago de Compostela.

Trask, R. L. (1993): A dictionary of grammatical terms in linguistics. London/ New York: Routledge.

Torres i Graell, A. (1983): Kanji. La escritura japonesa. Madrid: Hiperión.

Villacañas, J. L. (2019): Imperiofilia y el populismo nacional-católico, Madrid: Lengua de Trapo.

Wasow, T. (1997): "Remarks on grammatical weight". Language Variation and Change 9 (1): 81-105.

Whorf, B. L. (1956): Language, Thought, and Reality. Selected writings of Benjamin Lee Whorf, Cambridge, Mass.: Technology Press of Massachusetts Institute of Technology.

Yamashita, H., Chang, F. (2001): "Long before short preference in the production of a head-final language". Cognition 81 (2): B45-B55. 